\title{
Optical and structural properties of rice husk silicate incorporated borotellurite glasses doped with erbium oxide nanoparticles
}

\begin{abstract}
A system of erbium oxide nanoparticles doped rice-husk-silicate borotellurite glasses with compositional formula, $\{[(\mathrm{TeO} 2) 0.7$ (B2O3)0.3]0.8 (SiO2)0.2\}1-y (Er2O3 NPs)y with $\mathrm{y}=0.01,0.02,0.03,0.04$ and 0.05 was fabricated using the method of melt-quenching. Various characterizations and measurement techniques such as density and molar volume, X-ray diffraction, Fourier transform infrared, transmission electron microscopy and UV-Vis spectroscopy were carried out to study the structural, morphological and optical properties of the fabricated glasses. The density values for the glasses increased from 4.1900 to $4.6003 \mathrm{~g}$ $\mathrm{cm}-3$ with the addition of $1 \%$ to $5 \%$ of Er2O3 NPs in the glass structure. The increase can be ascribed to increase in the overall molar weight of the glass caused by the higher molecular weight of erbium. The microstructural nature revealed in the glass morphological evidence of nanoparticle agglomerations in the glasses. From the UV-Vis spectra both direct and indirect energy band gap decreased with Er2O3 NPs increased 0.01 to $0.02 \mathrm{~mol}$ and then increased thereafter up to $0.05 \mathrm{~mol} \%$. The refractive index was found to be high, in the range of 2.6050 to 2.6794 , metallization criterion ranged from 0.3268 to 0.3414 which according to Berwal et al. (J Mol Struct 1127:636-644, 2017), is an indication that the glass has good optical nonlinearity and also suggests the glass has good potential for non-linear optical application. The molar polarizability ranged from 7.7041 to $7.4515 \AA 3$ while the optical transmission coefficient values ranged from 0.6551 to 0.6691 . The high refractive index, good metallization criterion, polarizability and transmission coefficient values suggest that the erbium oxide nanoparticles doped Rice husk silicate borotellurite glasses studied has some good potential for optical fibre amplifier application.
\end{abstract}

